\title{
Nonresonant Micromachined Gyroscopes With Structural Mode-Decoupling
}

\author{
Cenk Acar and Andrei M. Shkel, Associate Member, IEEE
}

\begin{abstract}
This paper reports a novel four-degrees-of-freedom (DOF) nonresonant micromachined gyroscope design concept that addresses two major MEMS gyroscope design challenges: eliminating the mode-matching requirement and minimizing instability and drift due to mechanical coupling between the drive and sense modes. The proposed approach is based on utilizing dynamical amplification both in the 2-DOF drive-direction oscillator and the 2-DOF sense-direction oscillator, which are structurally decoupled, to achieve large oscillation amplitudes without resonance. The overall 4-DOF dynamical system is composed of three proof masses, where second and third masses form the 2-DOF sense-direction oscillator, and the first mass and the combination of the second and third masses form the 2-DOF drive-direction oscillator. The frequency responses of the drive and sense direction oscillators have two resonant peaks and a flat region between the peaks. The device is nominally operated in the flat regions of the response curves belonging to the drive and sense direction oscillators, where the gain is less sensitive to frequency fluctuations. This is achieved by designing the drive and sense anti-resonance frequencies to match. Consequently, by utilizing dynamical amplification in the decoupled 2-DOF oscillators, increased bandwidth and reduced sensitivity to structural and thermal parameter fluctuations and damping changes are achieved, leading to improved robustness and long-term stability over the operating time of the device.
\end{abstract}

Index Terms-Decoupled modes, disturbance rejection, inertial MEMS, micromachined gyroscopes, rate sensors.

\section{INTRODUCTION}

$\mathbf{M}$ ICROMACHINED gyroscopes are projected to become a potential alternative to expensive and bulky conventional inertial sensors. With micromachining processes allowing mass-production of micromechanical systems on a chip together with their control and signal conditioning electronics, low-cost and microsized gyroscopes will provide high accuracy rotation measurements leading to an even broader application spectrum, ranging from advanced automotive safety systems and on-chip navigation systems to interactive consumer electronics [1], [2]. However, due to unfavorable effects of scaling, the current state of the art micromachined gyroscopes require an order of magnitude improvement in performance, stability, and robustness [4], [7].

The conventional micromachined rate gyroscopes operate on the vibratory principle of a two-degrees-of-freedom (DOF)

Manuscript received October 2, 2001; revised December 23, 2002. The associate editor coordinating the review of this paper and approving it for publication was Dr. Ewald Benes.

The authors are with the Department of Mechanical and Aerospace Engineering, Microsystems Laboratory, University of California at Irvine, Irvine, CA 92697 USA (e-mail: cacar@uci.edu; ashkel@uci.edu).

Digital Object Identifier 10.1109/JSEN.2003.815799 system with a single proof mass suspended by flexures anchored to the substrate, which allow the mass to oscillate in two orthogonal directions, namely the drive and the sense directions. The proof mass is sustained in resonance in the drive direction, and in the presence of an angular rotation, the Coriolis force proportional to the input angular rate, is induced, exciting the proof mass in the sense direction. To achieve high sensitivity, the drive and the sense resonant frequencies are typically designed and tuned to match, and the device is controlled to operate at or near the peak of the response curve [8]. To enhance the sensitivity further, the device is packaged in high vacuum, minimizing energy dissipation due to viscous effects of air surrounding the mechanical structure.

Extensive research has been focused on design of symmetric suspensions and resonator systems for the mode-matching and minimizing temperature dependence [16], [17]. However, especially for lightly-damped devices, the requirement for modematching is well beyond fabrication tolerances, and none of the symmetric designs can provide the required degree of modematching without active tuning and feedback control [5], [6]. Furthermore, the mechanical interference between the modes, and thus the operation instability and drift, are proportional to the degree of mode-matching. Various devices have been proposed employing independent flexures for driving and sensing mode oscillations to suppress coupled oscillation and the resulting zero-rate drift [10]-[14], [16].

In this paper, we propose a novel 4-DOF micromachined gyroscope system utilizing dynamical amplification to achieve large oscillation amplitudes without resonance, while mechanically decoupling the drive direction oscillations from the sense direction oscillations. The overall 4-DOF dynamical system is composed of three proof masses (Fig. 1). The 2-DOF sense-direction oscillator is made up of the second and third masses, designed to amplify response in the sense-mode. The first mass and the combination of the second and third masses form the 2-DOF drive-direction oscillator. The drive and sense direction oscillators are mechanically decoupled, minimizing instability due to dynamical coupling between the drive and sense modes. The frequency response of both of the drive and sense direction oscillators have two resonant peaks and a flat region between the peaks. By designing the drive and sense antiresonance frequencies to match, the flat-region frequency band of the oscillators are overlapped, defining the nominal operation region of the device, where the response gain is less sensitive to parameter variations. Utilizing dynamical amplification in the 2-DOF oscillators instead of resonance, increased bandwidth and reduced sensitivity to structural and thermal parameter fluctuations and damping changes are achieved. The improved 


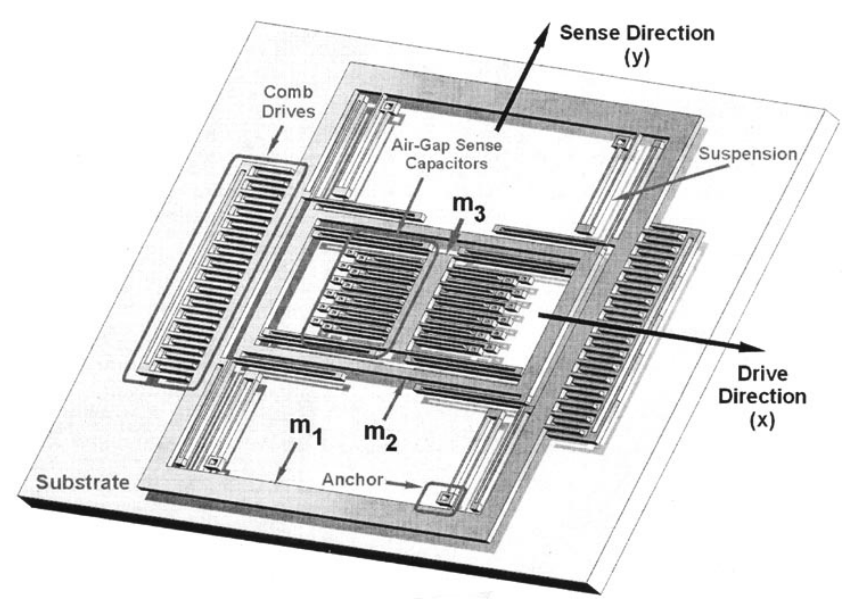

(a)

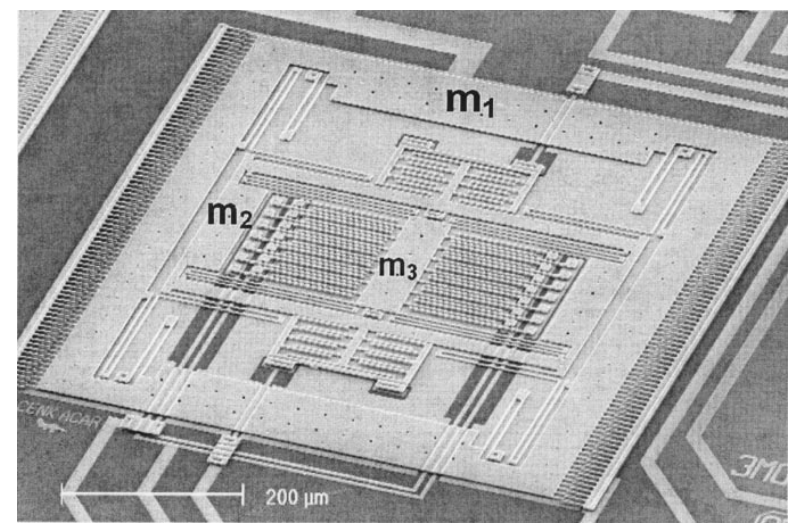

(b)

Fig. 1. (a) Conceptual schematic of the micromachined 4-DOF gyroscope with decoupled oscillation modes. (b) Scanning electron micrograph of the fabricated prototype 4-DOF gyroscope.

robustness and long-term stability over the operating time of the device is expected to relax control system requirements and tight fabrication and packaging tolerances.

The paper is organized as follows. We first present, in Section II, a detailed analysis of the conventional gyroscope dynamics emphasizing the related challenges. In Section III, the design approach and the principle of operation are presented, together with a detailed comparison of the 4-DOF system characteristics to a conventional gyroscope. The dynamics of the device is then analyzed in Section IV, and a MEMS implementation of the design concept is presented in Section V, along with an approach for determining optimal system parameters to maximize sensor performance.

\section{CONVENTIONAL Gyroscope APPROACH}

Almost all existing micromachined rate gyroscopes operate on the principle of rotation-induced Coriolis acceleration detection using a single vibrating proof mass suspended above the substrate. The proof mass is supported by anchored flexures, which serve as the flexible suspension between the proof mass and the substrate, making the mass free to oscillate in two orthogonal directions [Fig. 2(a)]: the drive direction ( $x$-axis) and the sense direction ( $y$-axis). The overall dynamical system is simply a 2-DOF mass-spring-damper system, where the drive direction is excited by the sinusoidal electrostatic force, and the sense direction is excited by the rotation-induced Coriolis force [2], [8].

If the motion of a conventional single-mass gyroscope is decomposed into the two principle oscillation directions, the drive direction $x$ and the sense direction $y$, the two equations of motion can be expressed as

$$
\begin{aligned}
& m \ddot{x}+c_{x} \dot{x}+\left(k_{x}-m\left(\Omega_{y}{ }^{2}+\Omega_{z}{ }^{2}\right)\right) x+m\left(\Omega_{x} \Omega_{y}-\dot{\Omega}_{z}\right) y \\
& \quad=F_{d}+2 m \Omega_{z} \dot{y} \\
& m \ddot{y}+c_{y} \dot{y}+\left(k_{y}-m\left(\Omega_{x}{ }^{2}+\Omega_{z}{ }^{2}\right)\right) y+m\left(\Omega_{x} \Omega_{y}+\dot{\Omega}_{z}\right) x \\
& \quad=-2 m \Omega_{z} \dot{x}
\end{aligned}
$$

where $m$ is the proof mass, $F_{d}$ is the drive direction excitation force, and $\vec{\Omega}=\left\{\Omega_{x}, \Omega_{y}, \Omega_{z}\right\}$ is the input angular velocity vector. The two final terms $2 m \Omega_{z} \dot{y}$ and $2 m \Omega_{z} \dot{x}$ are the rotationinduced Coriolis forces, causing dynamic coupling between the oscillation axes proportional to the angular rate input. In most of the reported micromachined vibratory rate gyroscopes, the proof mass is driven at or near the resonance frequency in the drive direction by an external sinusoidal force, which are generally the electrostatic forces applied by comb-drive structures [19]. When the gyroscope is subjected to an angular rotation, the Coriolis force with the same frequency as the driving signal is induced in the $y$-direction. If the drive and sense resonant frequencies are matched, the Coriolis force excites the system into resonance in the sense direction, as well. The resulting oscillation amplitude in the sense direction is proportional to the Coriolis force and, thus, to the angular velocity to be measured.

The dynamics of the single-mass 2-DOF gyroscope is understood more clearly starting with the assumption that the system is driven without feedback control in drive direction $x$ with a constant amplitude drive force $F_{d}$ at the drive frequency $\omega_{d}$, namely $F_{d}=F_{o} \sin \omega_{d} t$. The 2-DOF dynamical system will have two independent resonant frequencies: sense direction resonant frequency $\omega_{y}=\sqrt{k_{y} / m}$ and drive direction resonant frequency $\omega_{x}=\sqrt{k_{x} / m}$.

The rotation-induced Coriolis force $F_{c}=2 m \Omega_{z} \dot{x}$, which is proportional to drive direction oscillation amplitude is the only driving force in the sense direction for an ideal gyroscope. Sense direction amplitude is proportional to the Coriolis force $F_{c}$ and, thus, to drive direction oscillation amplitude. If the proof mass is driven into resonance in drive direction, i.e., $\omega_{d}=\omega_{x}$, a high sense direction amplitude is expected due to the increased Coriolis force resulting from large drive direction amplitudes achieved by resonance. If the system is driven at the sense direction resonant frequency, i.e., $\omega_{d}=\omega_{y}$, then the sense direction amplitude is amplified by the quality factor due to resonance. Thus, when the resonance frequencies are mismatched $\left(\omega_{x} \neq \omega_{y}\right)$, the frequency response of the 2-DOF system has two resonant peaks, one at $\omega_{x}$ and another at $\omega_{y}$. When the resonant frequencies are tuned to match exactly, i.e., $\omega_{x}=\omega_{y}$, the frequency response of the 2-DOF system has one combined resonant peak, which will provide a much larger response amplitude (Fig. 3), leading to the highest sensitivity possible.

However, the mode-matching requirement renders the system response very sensitive to variations in system parameters due 


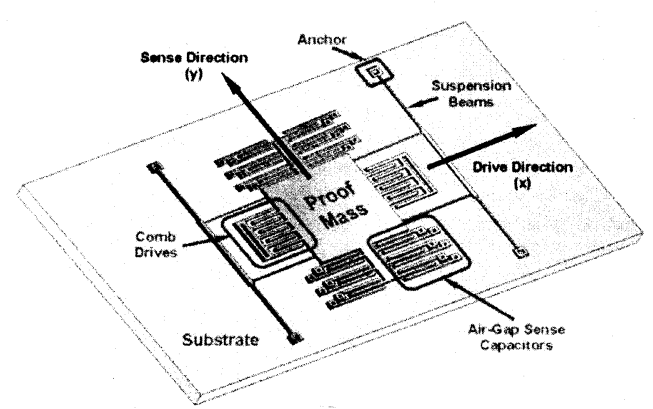

(a)

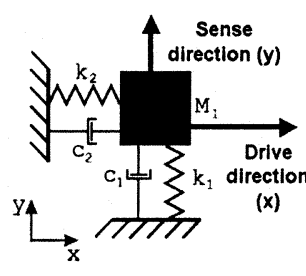

(b)

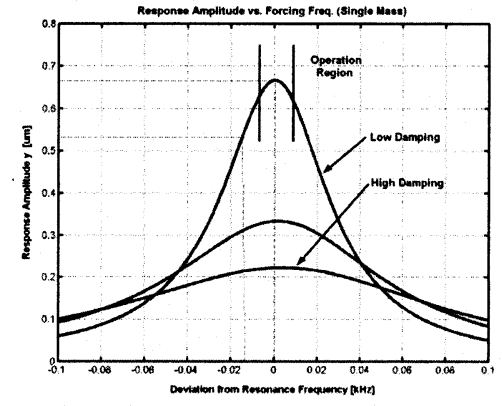

(c)

Fig. 2. (a) Schematic of the conventional 2-DOF gyroscope dynamical model. (b) A typical MEMS implementation of a conventional 2-DOF gyroscope. (c) The response in the sense direction excited by the Coriolis force, with variation in damping conditions.

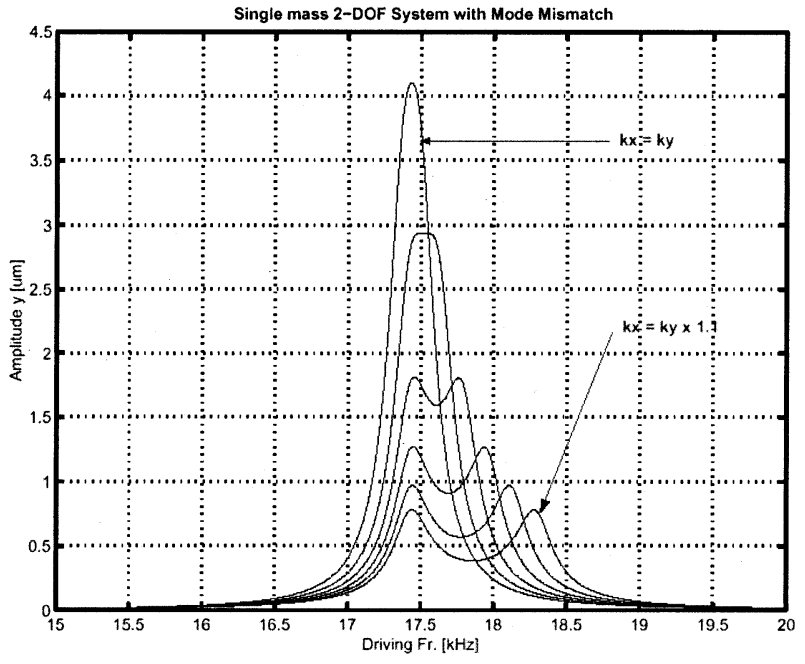

Fig. 3. Response of the overall 2-DOF system, which diminishes with increasing drive and sense resonance frequency mismatch.

to fabrication imperfections and fluctuations in operating conditions, which shift the drive or sense resonant frequencies. Inevitable fabrication imperfections affect both the geometry and the material properties of MEMS devices [7]. The designed stiffness values deviate drastically due to etching processes, deposition conditions, or residual stresses. Variations in the temperature of the structure can also perturb the dynamical system parameters due to the temperature dependence of Young's Modulus and thermally induced localized stresses.

Various symmetric gyroscope designs have been reported based on enhancing performance by mode-matching and allowing to minimize temperature dependence [16], [17]. However, mode-matching requirements cannot be met without feedback control, even with the symmetric designs under the presence of the mentioned perturbations. For the devices packaged in high vacuum to enhance the sensitivity, the bandwidth of the resonance peaks is extremely narrow, leading to much tighter mode-matching requirements [5]. Also the gain is affected significantly by fluctuations in damping conditions [Fig. 2(c)], which makes the device very vulnerable to any possible vacuum leak in the package. Furthermore, as the modes are matched closer, the mechanical interference between the modes becomes more significant, resulting in operation instability and drift. In order to suppress coupled oscillation and drift, various devices have been reported employing independent suspension beams for the drive and sense modes [13]-[17].

The 4-DOF gyroscope design concept proposed in this paper eliminates the limitations due to mode-matching requirement, damping sensitivity and coupled oscillation challenges by utilizing mechanically decoupled 2-DOF nonresonant drive and sense oscillators incorporating three proof masses. 4-DOF MEMS gyroscopes composed of two interconnected proof masses have been reported to achieve improved robustness [9]; however, the drive and sense oscillators cannot be mechanically decoupled, and the dynamical response characteristics of the oscillators can't be set independently in these approaches.

\section{4-DOF GYROSCOPE STRUCTURE AND PRINCIPLE OF OPERATION}

In this paper, we propose a novel 4-DOF micromachined gyroscope system that utilizes dynamical amplification in the decoupled 2-DOF drive and sense oscillators in order to achieve large oscillation amplitudes without resonance. The overall 4-DOF dynamical system, namely 2-DOF in drive and 2-DOF in sense directions, is composed of three interconnected proof masses (Fig. 1).

The first mass $m_{1}$, which is the only mass excited in the drive direction, is constrained in the sense direction, and is free to oscillate only in the drive direction. The second mass $m_{2}$ and third mass $m_{3}$ are constrained with respect to each other in the drive direction, thus oscillating as one combined mass in the drive direction. However, $m_{2}$ and $m_{3}$ are free to oscillate independently in the sense direction, forming the 2-DOF sense-direction oscillator. The first mass $m_{1}$ and the combination of the second and third masses $\left(m_{2}+m_{3}\right)$ form the 2-DOF drive-direction oscillator, where $m_{1}$ is the driven mass (Fig. 4).

In order to minimize instability due to dynamical coupling between the drive and sense modes, the drive and sense direction oscillators are mechanically decoupled. The driven mass $m_{1}$ oscillates only in the drive direction, and possible anisoelasticities due to fabrication imperfections are suppressed by the suspension fixed in the sense direction. The second mass $m_{2}$ oscillates in both drive and sense directions, and generates the rotation-induced Coriolis force that excites the 2-DOF sense-direction oscillator. The sense direction response of the third mass $m_{3}$, 


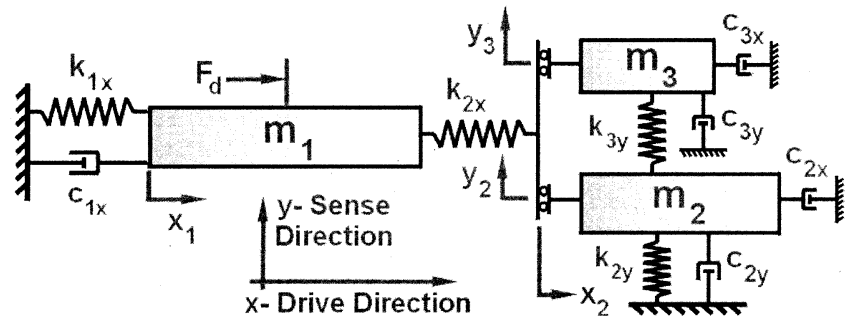

Fig. 4. Lumped mass-spring-damper model of the overall 4-DOF gyroscope dynamical system.

which comprises the vibration absorber of the 2-DOF sense-direction oscillator, is detected for measuring the input angular rate. Since the springs that couple the sense element $m_{3}$ to $m_{2}$ deform only for relative sense direction oscillations, instability due to mechanical coupling of drive and sense directions is minimized, significantly enhancing gyroscopic performance due to reduced drift.

\section{A. Coriolis Response}

The frequency responses of the 2-DOF drive direction oscillator and the 2-DOF sense direction oscillator have two resonant peaks and a flat region between the peaks. The device is nominally operated in the flat regions of the drive and sense direction oscillators, where the response amplitudes of the oscillators are less sensitive to parameter variations. In order to operate both of the drive and sense direction oscillators in their flat-region frequency bands, the flat regions of the oscillators have to be designed to overlap [Fig. 5(a)] by matching the drive and sense direction anti-resonance frequencies, as will be explained in Section V-C. However, in contrast to the conventional gyroscopes, the flat regions with significantly wider bandwidths can be overlapped without feedback control with sufficient precision in spite of fabrication imperfections and operation condition variations.

The response of the combined 4-DOF dynamical system to the rotation-induced Coriolis force will have a flat region in the frequency band coinciding to the flat regions of the independent drive and sense-mode oscillators [Fig. 5(b)]. When the device is operated in this flat region, the oscillation amplitudes in both drive and sense directions are relatively insensitive to variations in system parameters and damping. Thus, by utilizing dynamical amplification in the 2-DOF oscillators instead of resonance, increased bandwidth and reduced sensitivity to structural and thermal parameter fluctuations and damping changes are achieved. Consequently, the design concept resulting in improved robustness and long-term stability over the operating time of the device is expected to relax control requirements and tight fabrication and packaging tolerances.

\section{B. Comparison of Response Characteristics With a Conventional Gyroscope}

The proposed design approach allows to widen the operation frequency range of the gyroscope to achieve improved robustness, while sacrificing the response amplitude in the sense direction. The following numerical example demonstrates the trade-off between bandwidth of the gyroscope and the amplitude of response. For a clear comparison, we consider a conventional 2-DOF gyroscope with the same mass, stiffness and damping parameters as the isolated passive mass-spring system of the 4-DOF system, which is designed with matched resonance frequencies at $10.3 \mathrm{kHz}$ in the drive and sense directions. It is assumed that both gyroscopes are vacuum packaged so that the pressure within the encapsulated cavity is equal to 100 miliTorrs $(13.3 \mathrm{~Pa})$, and are both excited to achieve $5-\mu \mathrm{m}$ drive-direction oscillation amplitudes. Under these conditions, for $1 \%$ s input angular rate, the conventional 2-DOF gyroscope will have a $2.8 \times 10^{-3} \mu \mathrm{m}$ response amplitude in the sense direction, while the sense mass of the 4-DOF gyroscope will have an amplitude of response equal to $0.72 \times 10^{-3} \mu \mathrm{m}$. However, in the case of the conventional 2-DOF gyroscope, the extremely narrow bandwidth of the response (about $1.1 \mathrm{~Hz}$ ) significantly limits the robustness of the device. In a 2-DOF system, a frequency mismatch of $0.1 \%$ between drive and sense directions causes drop of the sense-direction amplitude down to $1.15 \times 10^{-3} \mu \mathrm{m}$ (59\% gain drop), and $1 \%$ frequency mismatch results in $0.14 \times 10^{-3} \mu$ m oscillation amplitude $(94 \%$ gain drop). In contrast, the 4-DOF gyroscope demonstrates significantly improved robustness against system parameter variations; $1 \%$ deviation in passive mass resonance frequency results in $0.70 \times 10^{-3} \mu \mathrm{m}$ amplitude (3\% change in the gain). It should be also noted that, if the pressure is increased inside the package by $10 \%$, it will lead to a $14 \%$ drop in gain of the conventional gyroscope, while the gain of the 4-DOF gyroscope will change by less than $0.1 \%$.

Moreover, since the design space of the 4-DOF gyroscope is larger than of the conventional gyroscope, the design concept allows maximum flexibility in optimizing the system response. The optimal compromise between amplitude of the response and bandwidth can be obtained by selecting parameters of the system (ratio of masses and spring constants). For example, the gain of the 4-DOF system can be improved by trading-off bandwidth; if the coupling spring constant between the passive and active masses of the 4 DOF gyroscope is increased from $4.2 \mathrm{~N} / \mathrm{m}$ to $4.8 \mathrm{~N} / \mathrm{m}$, the amplitude of the response in the sense direction will increase from $0.72 \times 10^{-3} \mu \mathrm{m}$ to $1.2 \times 10^{-3} \mu \mathrm{m}$, while the response bandwidth will decrease from $23 \mathrm{~Hz}$ to $12 \mathrm{~Hz}$, which is still over an order of magnitude larger than the bandwidth of the conventional gyroscope discussed above. Thus, the 4-DOF design concept provides more freedom in defining trade-offs between gain of the response (for higher sensitivity) and the system bandwidth (for increased robustness). Selection of the parameter set is typically guided by application requirements.

\section{DYNAMICS OF THE GYROSCOPE}

The dynamics of the idealized model for the 4-DOF gyroscope system is best understood in the noninertial coordinate frame associated with the gyroscope. The 4-DOF system consists of three interconnected proof masses where each mass can be assumed to be a rigid body with a position vector $\vec{r}$ attached to a rotating reference frame $B$, resulting in an absolute acceleration in the inertial frame $A$

$$
\overrightarrow{a_{A}}=\overrightarrow{a_{B}}+\overrightarrow{\vec{\Omega}} \times \overrightarrow{r_{B}}+\vec{\Omega} \times\left(\vec{\Omega} \times \overrightarrow{r_{B}}\right)+2 \vec{\Omega} \times \overrightarrow{v_{B}}
$$




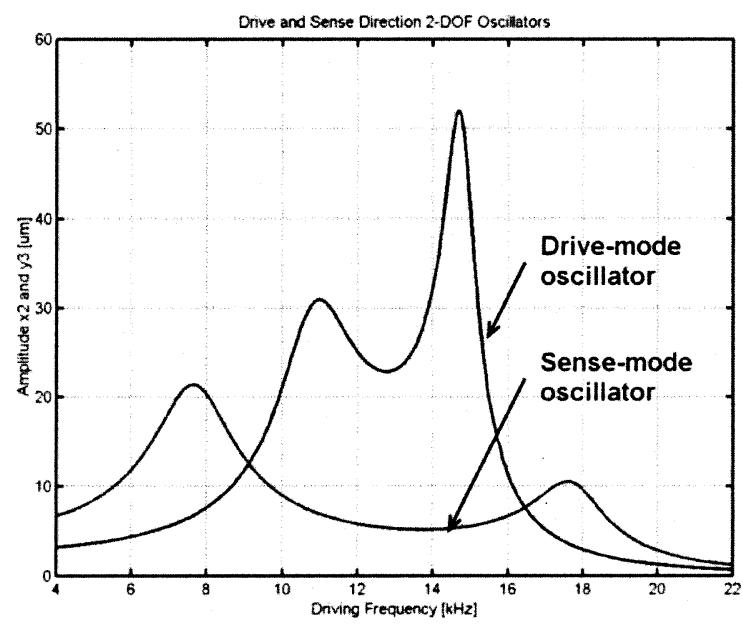

(a)

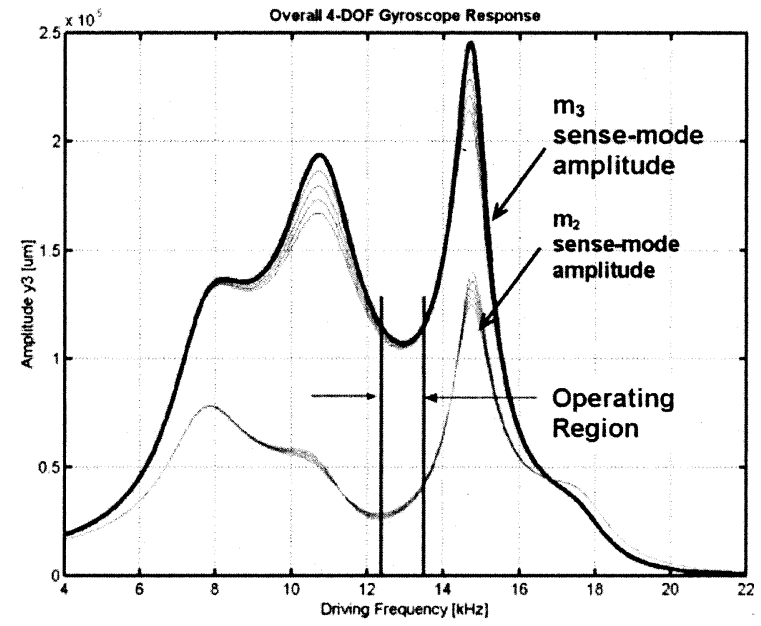

(b)

Fig. 5. (a) Frequency responses of the 2-DOF drive and sense-mode oscillators, with the overlapped flat regions. (b) The response of the overall 4-DOF gyroscope system. The oscillation amplitude is relatively insensitive to parameter variations and damping fluctuations in the flat operating region.

where the subscript $A$ denotes "relative to inertial frame $A$," denotes "relative to rotating gyroscope frame $B, \overrightarrow{v_{B}}$ and $\overrightarrow{a_{B}}$ are the velocity and acceleration vectors with respect to the reference frame $B$, respectively, and $\vec{\Omega}$ is the angular velocity of the gyroscope frame $B$ relative to the inertial frame $A$. The term $2 \vec{\Omega} \times \overrightarrow{v_{B}}$ is the Coriolis acceleration, and excites the system in the sense direction. Thus, when a mass oscillating in the drive direction ( $x$-axis) is subject to an angular rotation rate of $\Omega_{z}$ about the $z$-axis, the Coriolis acceleration induced in the sense direction ( $y$-axis) is $a_{y}=2 \Omega_{z} \dot{x}(t)$.

Similarly, the equations of motion for the three proof masses observed in the noninertial rotating frame can be expressed in the inertial frame as

$$
\begin{aligned}
m_{1} \overrightarrow{a_{1}}= & \overrightarrow{F_{1}}+\overrightarrow{F_{d}}-2 m_{1} \vec{\Omega} \times \overrightarrow{v_{1}}-m_{1} \vec{\Omega} \\
& \times\left(\vec{\Omega} \times \overrightarrow{r_{1}}\right)-m_{1} \dot{\vec{\Omega}} \times \overrightarrow{r_{1}} \\
m_{2} \overrightarrow{a_{2}}= & \overrightarrow{F_{2}}-2 m_{2} \vec{\Omega} \times \overrightarrow{v_{2}}-m_{2} \vec{\Omega} \\
& \times\left(\vec{\Omega} \times \overrightarrow{r_{2}}\right)-m_{2} \dot{\vec{\Omega}} \times \overrightarrow{r_{2}} \\
m_{3} \overrightarrow{a_{3}}= & \overrightarrow{F_{3}}-2 m_{3} \vec{\Omega} \times \overrightarrow{v_{3}}-m_{3} \vec{\Omega} \\
& \times\left(\vec{\Omega} \times \overrightarrow{r_{3}}\right)-m_{3} \dot{\vec{\Omega}} \times \overrightarrow{r_{3}}
\end{aligned}
$$

where $\vec{F}_{1}$ is the net external force applied to $m_{1}$ including elastic and damping forces from the substrate and elastic interaction force from $m_{2} ; \vec{F}_{2}$ is the net external force applied to $m_{2}$ including the damping force from the substrate and elastic interaction force from $m_{1}$ and $m_{3} ; \vec{F}_{3}$ is the net external force applied to $m_{3}$ including the damping force from the substrate and the elastic interaction force from $m_{2}$, and $\vec{F}_{d}$ is the driving force applied to $m_{1}$. In the gyroscope frame, $\overrightarrow{r_{1}}, \overrightarrow{r_{2}}$, and $\overrightarrow{r_{3}}$ are the position vectors, and $\overrightarrow{v_{1}}, \overrightarrow{v_{2}}$, and $\overrightarrow{v_{3}}$ are the velocity vectors of $m_{1}$, $m_{2}$, and $m_{3}$, respectively. Since the first mass is fixed in the sense direction [i.e., $y_{1}(t)=0$, and $m_{2}$ and $m_{3}$ move together in the drive direction i.e., $\left.x_{2}(t)=x_{3}(t)\right]$, the 4-DOF equations of motion (along the $x$-axis and $y$-axis) of the three mass systems subjected to an angular rate of $\Omega_{z}$ about the axis normal to the plane of motion ( $z$-axis) become

$$
\begin{aligned}
& m_{1} \ddot{x_{1}}+c_{1 x} \dot{x_{1}}+k_{1 x} x_{1} \\
& \quad=k_{2 x}\left(x_{2}-x_{1}\right)+m_{1} \Omega_{z}^{2} x_{1}+F_{d}(t) \\
& \left(m_{2}+m_{3}\right) \ddot{x_{2}}+\left(c_{2 x}+c_{3 x}\right) \dot{x_{2}}+k_{2 x}\left(x_{2}-x_{1}\right) \\
& \quad=\left(m_{2}+m_{3}\right) \Omega_{z}^{2} x_{2}+2 m_{2} \Omega_{z} \dot{y_{2}}+2 m_{3} \Omega_{z} \dot{y_{3}} \\
& \quad+m_{2} \dot{\Omega}_{z} y_{2}+m_{3} \dot{\Omega}_{z} y_{3} \\
& m_{2} \ddot{y_{2}}+c_{2 y} \dot{y_{2}}+k_{2 y} y_{2} \\
& \quad=k_{3 y}\left(y_{3}-y_{2}\right)+m_{2} \Omega_{z}^{2} y_{2}-2 m_{2} \Omega_{z} \dot{x_{2}}-m_{2} \dot{\Omega}_{z} x_{2} \\
& m_{3} \ddot{y_{3}}+c_{3 y} \dot{y_{3}}+k_{3 y}\left(y_{3}-y_{2}\right) \\
& \quad=m_{3} \Omega_{z}^{2} y_{3}-2 m_{3} \Omega_{z} \dot{x_{3}}-m_{3} \dot{\Omega}_{z} x_{3}
\end{aligned}
$$

where $F_{d}(t)$ is the driving electrostatic force applied to the active mass at the driving frequency $\omega_{d}$, and $\Omega_{z}$ is the angular velocity applied to the gyroscope about the $z$-axis. It should be noted that the terms $2 m_{2} \Omega_{z} \dot{x_{2}}$ and $2 m_{3} \Omega_{z} \dot{x_{3}}$ are the Coriolis forces that excite the system in the sense direction, and the Coriolis response of $m_{3}$ in the sense-direction $\left(y_{3}\right)$ is detected for angular rate measurement.

\section{MEMS IMPLEMENTATION OF THE DESIGN CONCEPT}

This section describes the principle elements of a MEMS implementation of the conceptual design presented in Section III. First, the suspension system design for the 4-DOF system is investigated with the derivation of the stiffness values, then the damping components of the dynamic system derived in Section IV are analyzed. Finally, the issue of achieving dynamic amplification in the drive and sense modes is addressed, along with an approach for determining optimal system parameters to maximize sensor performance.

\section{A. Suspension Design}

The complete suspension system of the device is designed such that the first mass $m_{1}$ with 1-DOF is fixed in the sense direction, and is free to oscillate only in the drive direction, the second mass $m_{2}$ has 2-DOF, oscillating in both drive and sense 


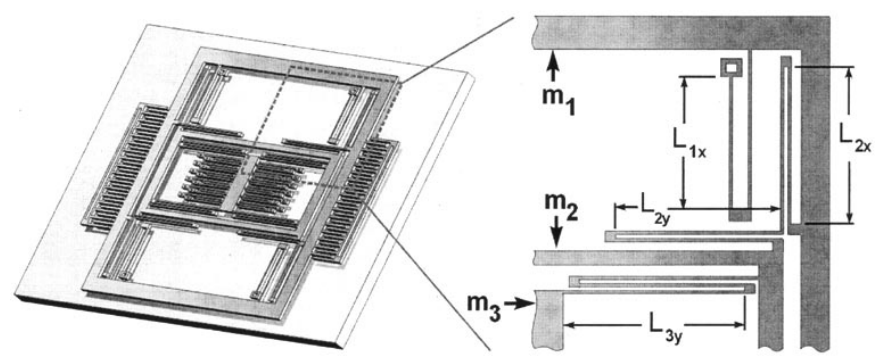

Fig. 6. The suspension system configuration that forms the mechanically decoupled 2-DOF drive and sense direction oscillators with the three proof masses.

directions, and the third mass $m_{3}$ with 1-DOF is fixed with respect to $m_{2}$ in the drive direction, and free to oscillate independently in the sense direction (Fig. 6).

The suspension that connects $m_{1}$ to the substrate via anchors is comprised of four double-folded flexures, where each beam of length $L_{1 x}$ in the folded flexures can be modeled as a fixedguided beam deforming in the orthogonal direction to the axis of the beam, leading to an overall stiffness of [21]

$$
k_{1 x}=\frac{4}{2}\left(\frac{1}{2} \frac{3 E I}{\frac{L_{1 x}}{2}}\right)=\frac{2 E t w^{3}}{L_{1 x}^{3}}
$$

where $E$ is the Young's Modulus, $I$ is the second moment of inertia of the beam cross-section, $t$ is the beam thickness, and $w$ is the beam width. Possible anisoelasticities due to fabrication imperfections are suppressed having the driven mass $m_{1}$ oscillating purely along the geometrical drive axis by this suspension constraining $m_{1}$ in the sense direction. Effects of residual stresses are also decreased by employing folded springs allowing stress-relief.

The second mass $m_{2}$ is connected to $m_{1}$ by four flexures composed of two double-folded beams of length $L_{2 x}$ and $L_{2 y}$ that deform independently in the drive and sense directions. These beams can also be modeled similarly, resulting in $m_{2}$ drive and sense direction stiffness values of

$$
k_{2 x}=\frac{2 E t w^{3}}{L_{2 x}{ }^{3}}, \quad k_{2 y}=\frac{2 E t w^{3}}{L_{2 y}{ }^{3}} .
$$

In the calculation of the spring rates in the drive or sense direction, the effect of axial strain in the other beams is neglected. The assumption is reasonable in this analysis, since the axial stiffness of a beam, $k_{\text {axial }}=E t w / L$, is generally four orders of magnitude ( $L^{2} / w^{2}$ times) larger than the fixed-guided stiffness, which means the beams under axial load can be assumed infinitely stiff.

The suspension connecting the third mass $m_{3}$ to $m_{2}$ is made up of four three-folded flexures for this specific design, fixing $m_{3}$ with respect to $m_{2}$ in the drive-direction. Since these flexures are stiff in the drive-direction and deform only in the sensedirection, instability due to dynamical coupling between the drive and sense modes in the sensing element $m_{3}$ is eliminated, minimizing zero-rate drift of the gyroscope. With a length of $L_{3 y}$ for each beam, the overall stiffness is

$$
k_{3 y}=\frac{4}{3} \frac{E t w^{3}}{L_{3 y}{ }^{3}} .
$$

The lengths $L_{1 x}, L_{2 x}, L_{2 y}$, and $L_{3 y}$ are designed according to the optimized stiffness values derived in Section V-C. Through this suspension design, mechanically decoupled 2-DOF drive and sense direction oscillators are formed with the three proof masses, while instability due to mechanical coupling of drive and sense directions is minimized, leading to significantly reduced zero-rate drift [16].

\section{B. Damping Estimation}

The dominant mechanism of energy dissipation in the gyroscope structure is the internal friction of the fluid confined between the proof mass surfaces and the stationary surfaces. The damping coefficients $c_{1 x}, c_{2 x}, c_{2 y}$, and $c_{3 y}$ in the gyroscope dynamical system shown in Fig. 4 are due to the viscous effects of the air between the masses and the substrate, and in between the comb-drive and sense capacitor fingers.

For the driven mass $m_{1}$, the total damping in the drive mode can be approximated as the combination of the slide film damping between the mass and the substrate, and the slide film damping between the integrated comb fingers. Assuming an instantaneously developed linear fluidic velocity profile, slide film damping can be modeled as a Couette flow, leading to

$$
c_{1 x}=\mu_{e} \frac{A_{1}}{z_{0}}+\mu_{e} \frac{2 N_{\mathrm{comb}} l_{\mathrm{comb}} t}{y_{\mathrm{comb}}}
$$

where $A_{1}$ is the area of the active mass, $z_{0}$ is the elevation of the proof mass from the substrate, $t$ is the thickness of the structure, $N_{\text {comb }}$ is the number of comb-drive fingers, $y_{\text {comb }}$ is the distance between the fingers, and $l_{\text {comb }}$ is the overlapping length of the fingers. The effective viscosity is $\mu_{e}=\mu_{p} p$, where $p$ is the ambient pressure within the cavity of the packaged device, and $\mu_{p}=3.710^{-4} \mathrm{~kg} / \mathrm{m}^{2}$.s.torr $\left(\mu_{p}=2.7810^{-6}\left[\left(\mathrm{~kg} / \mathrm{m}^{2}\right.\right.\right.$.s.Pa $\left.)\right]$ is the viscosity constant for air [8].

Since there are no actuation and sensing capacitors attached to the second mass $m_{2}$, the damping coefficients in the drive and sense directions are equal, and are only due to the Couette flow between the proof mass and the substrate

$$
c_{2 x}=c_{2 y}=\mu_{e} \frac{A_{2}}{z_{0}} .
$$

Thus, the approach results in reduced energy dissipation on $m_{2}$, and also symmetric damping in the drive and sense directions. For the third mass $m_{3}$, the total damping in the drive mode results from Couette flow between the mass and the substrate, as well as Couette flow between the air-gap capacitor fingers

$$
c_{3 x}=\mu_{e} \frac{A_{3}}{z_{0}}+\mu_{e} \frac{2 N_{\text {cap }} l_{\text {cap }} t}{y_{\text {cap }}}
$$

where $A_{3}$ is the area of the passive mass, $N_{\text {cap }}$ is the number of air-gap capacitors, $y_{\text {cap }}$ is the distance between the capacitor fingers, and $l_{\text {cap }}$ is the overlapping length of the fingers. Damping on $m_{3}$ in the sense mode can be estimated as the combination of Couette flow between the proof mass and the substrate, and the squeeze-film damping between the air-gap capacitor fingers:

$$
c_{3 y}=\mu_{e} \frac{A_{3}}{z_{0}}+\mu_{e} \frac{7 N_{\text {cap }} l_{\text {cap }} t^{3}}{y_{\text {cap }}^{3}} .
$$




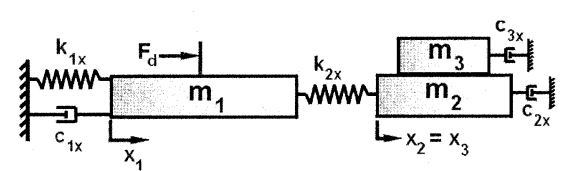

(a)

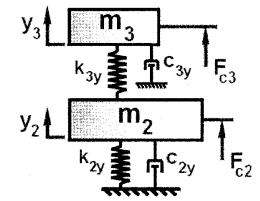

(b)
Fig. 7. (a) Lumped mass-spring-damper model for the 2-DOF drive-mode oscillator of the 4-DOF gyroscope. (b) Lumped mass-spring-damper model for the 2-DOF sense-mode oscillator.

It should be emphasized that the design approach suggests the sensing mass $m_{3}$ to have minimal mass in contrast to the conventional gyroscopes. This allows minimizing the overall energy dissipation on the sensing mass $m_{3}$ due to the reduced footstep area of the mass. More accurate slide-film damping models can be generated considering the gas-rarefaction effects at low pressures and narrow gaps [23], kinetic gas models, or plate motions that propagate into the fluid with rapidly diminishing steady-state amplitude [24]. Including the nonlinear effects of squeeze-film damping [25] together with computational fluid dynamics simulations will also improve the accuracy of the damping model.

\section{Parameter Optimization for Dynamic Amplification}

Since the foremost mechanical factor determining the performance of the gyroscope is the sense direction deflection of the sensing element $m_{3}$ due to the input rotation, the parameters of the dynamical system should be optimized to maximize the oscillation amplitude of $m_{3}$ in the sense direction.

However, the optimal compromise between amplitude of the response and bandwidth should be obtained to maintain robustness against parameters variations, while the response amplitude is sufficient for required sensitivity. The trade-offs between gain of the response (for higher sensitivity) and the system bandwidth (for increased robustness) will typically be guided by application requirements.

For the purpose of optimizing each parameter in the dynamical system, the overall 4-DOF gyroscope system can be decomposed into the 2-DOF drive direction oscillator [Fig. 7(a)] and the 2-DOF sense direction oscillator [Fig. 7(b)], to be analyzed separately in the Sections V-C-I and II.

1) Drive Mode Parameters: The first mass $m_{1}$ (which is free to oscillate only in the drive direction, and fixed in the sense direction) and the combination of the second and third masses $m_{2}+m_{3}$ (which are fixed with respect to each other in the drive direction) form the 2-DOF drive-direction oscillator, where $m_{1}$ is driven by the electrostatic forces. The main objective of pa- rameter optimization in the drive mode is to maximize the rotation-induced Coriolis force generated by the second mass $m_{2}$. This force $F_{c 2}=2 m_{2} \Omega_{z} \dot{x_{2}}$ is the dominant force exciting the 2-DOF sense-direction oscillator, and is proportional to the sensor sensitivity.

In the drive mode, the gyroscope is simply a 2-DOF system. The sinusoidal drive force is applied to the first mass $m_{1}$ (active mass) by the comb drive structures [19]. The combination of the second and the third masses $\left(m_{2}+m_{3}\right)$ comprise the vibration absorber (passive mass) of the 2-DOF oscillator, which mechanically amplifies the oscillations of $m_{1}$. Approximating the gyroscope by a lumped mass-spring-damper model [Fig. 7(a)], the equations of motion in the drive direction can be expressed as

$$
\begin{aligned}
m_{1} \ddot{x_{1}}+c_{1 x} \dot{x_{1}}+k_{1 x} x_{1} & =k_{2 x}\left(x_{2}-x_{1}\right)+F_{d} \\
\left(m_{2}+m_{3}\right) \ddot{x_{2}}+c_{2 x} \dot{x_{2}}+k_{2 x} x_{2} & =k_{2 x} x_{1} .
\end{aligned}
$$

When a constant-amplitude sinusoidal force $F_{d}=F_{0} \sin (\omega t)$ is applied on the active mass $m_{1}$ by the interdigitated comb-drives, the steady-state response of the 2-DOF system [Fig. 8(a)] will be as seen in the equation at the bottom of the page, where $\omega_{1 x}=\sqrt{k_{1 x} / m_{1}}$ and $\omega_{2 x}=\sqrt{k_{2 x} /\left(m_{2}+m_{3}\right)}$ are the resonant frequencies of the isolated active and passive mass-spring systems, respectively. When the driving frequency $\omega_{\text {drive }}$ is matched with the resonant frequency of the isolated passive mass-spring system, i.e., $\omega_{\text {drive }}=\sqrt{k_{2 x} /\left(m_{2}+m_{3}\right)}$, the passive mass moves to exactly cancel out the input force $F_{d}$ applied on the active mass, and maximum dynamic amplification is achieved [18].

Maximizing the Coriolis Force $F_{c 2}=2 m_{2} \Omega_{z} \dot{x_{2}}$ generated by $m_{2}$ requires a large proof mass $m_{2}$, and large drive direction amplitude $x_{2}$. However, if the response of the passive mass in the drive direction is observed for varying $m_{2}$ values with $m_{1}$ being fixed, it is seen that for high oscillation amplitudes of passive mass, $\left(m_{2}+m_{3}\right)$ should be minimized [Fig. 9(a)]. The anti-resonant frequency $\omega_{2 x}$ of the isolated passive massspring system is determined according to gyroscope operating frequency specifications, noting that larger Coriolis forces are induced at higher frequencies, but the oscillation amplitudes become larger at lower frequencies [Fig. 9(b)]. Once $\omega_{2 x}$ is fixed, the drive direction spring constant $k_{2 x}$ is obtained from $\omega_{2 x}$ and $\left(m_{2}+m_{3}\right)$.

The optimal drive direction mass ratio $\mu_{x}=\left(m_{2}+m_{3}\right) / m_{1}$ determining the mass of the active mass is dictated by low sensitivity to damping, response bandwidth and oscillation amplitude [18]. In order to achieve insensitivity to damping, the resonance peaks of the 2-DOF system response have to be separated

$$
\begin{aligned}
& X_{1}=\frac{F_{0}}{k_{1 x}} \frac{1-\left(\frac{\omega}{\omega_{2 x}}\right)^{2}+j \omega \frac{c_{2 x}}{k_{2 x}}}{\left[1+\frac{k_{2 x}}{k_{1 x}}-\left(\frac{\omega}{\omega_{1 x}}\right)^{2}+j \omega \frac{c_{1 x}}{k_{1 x}}\right]\left[1-\left(\frac{\omega}{\omega_{2 x}}\right)^{2}+j \omega \frac{c_{2 x}}{k_{2 x}}\right]-\frac{k_{2 x}}{k_{1 x}}} \\
& X_{2}=\frac{F_{0}}{k_{1}} \frac{1}{\left[1+\frac{k_{2 x}}{k_{1 x}}-\left(\frac{\omega}{\omega_{1 x}}\right)^{2}+j \omega \frac{c_{1 x}}{k_{1 x}}\right]\left[1-\left(\frac{\omega}{\omega_{2 x}}\right)^{2}+j \omega \frac{c_{2 x}}{k_{2 x}}\right]-\frac{k_{2 x}}{k_{1 x}}}
\end{aligned}
$$




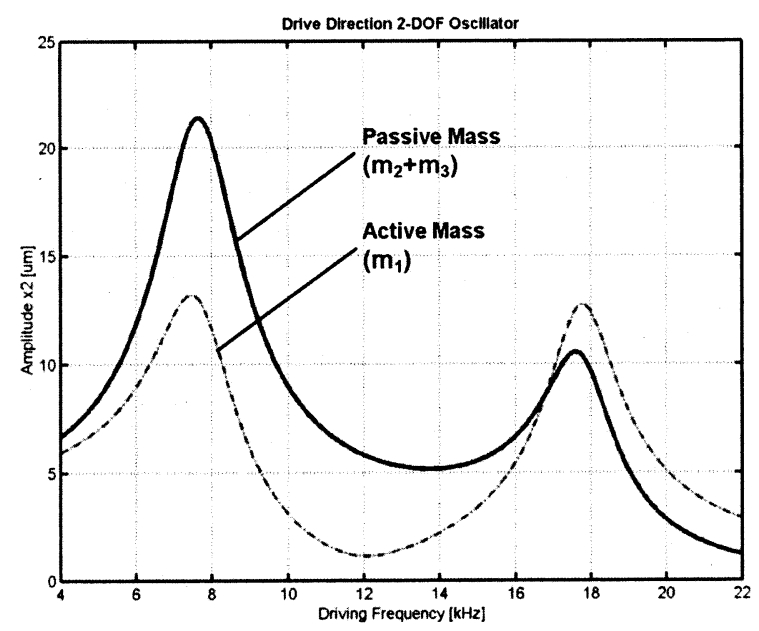

(a)

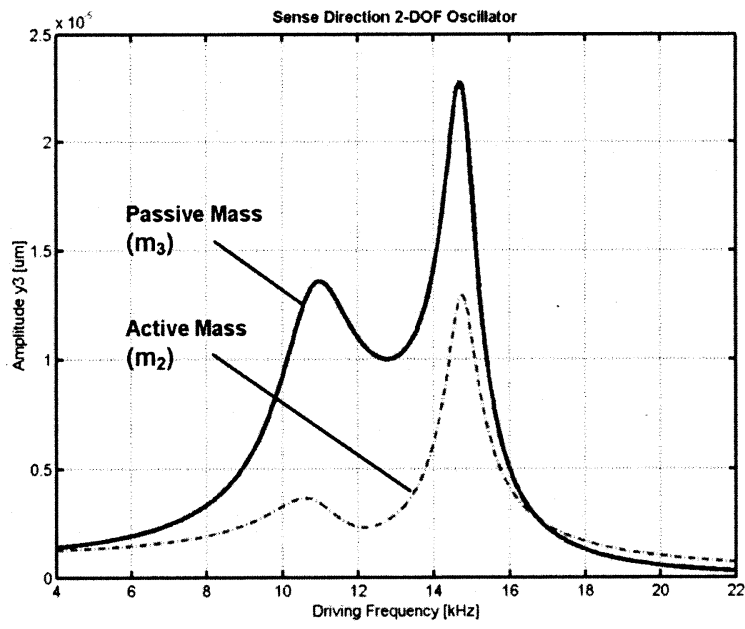

(b)

Fig. 8. (a) Frequency response of the 2-DOF drive-mode oscillator. (b) Frequency response of the 2-DOF sense-mode oscillator.

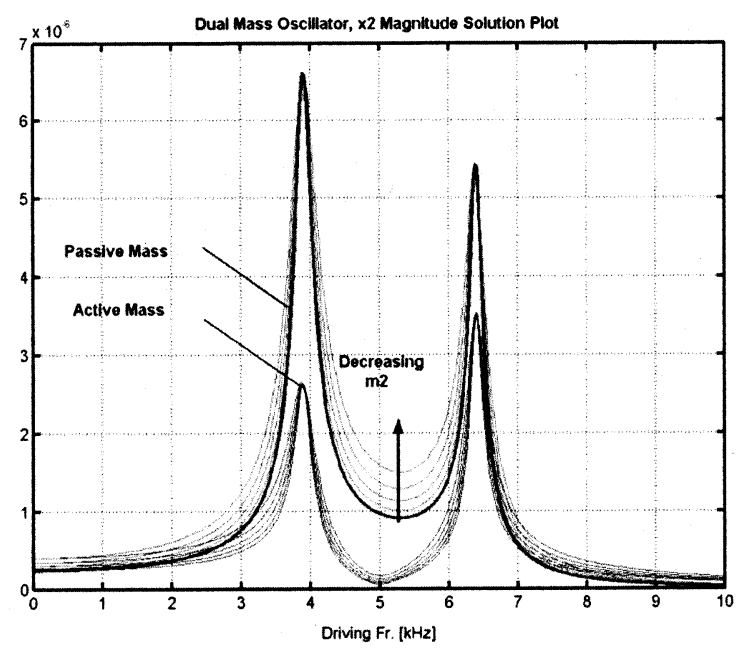

(a)

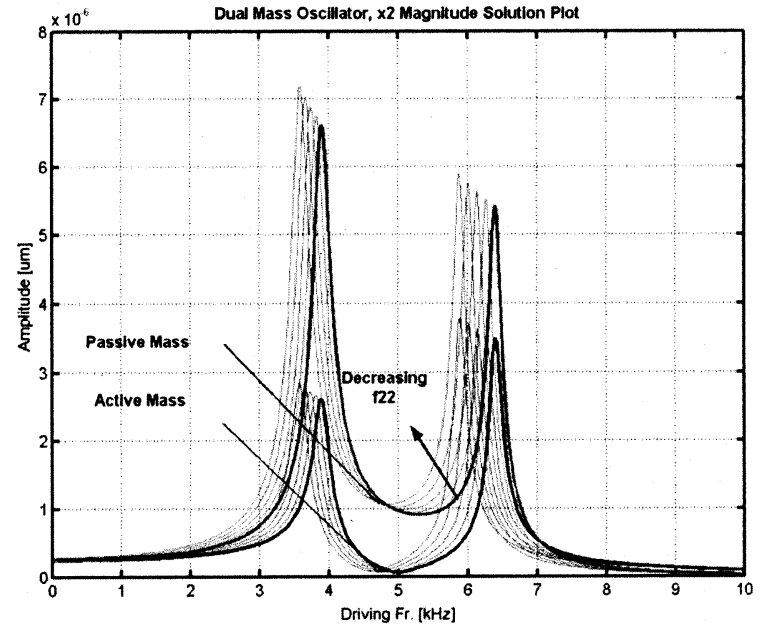

(b)

Fig. 9. Effect of (a) passive mass $\left(m_{2}+m_{3}\right)$ variation and (b) antiresonant frequency $\omega_{2 x}$ variation on drive direction response.

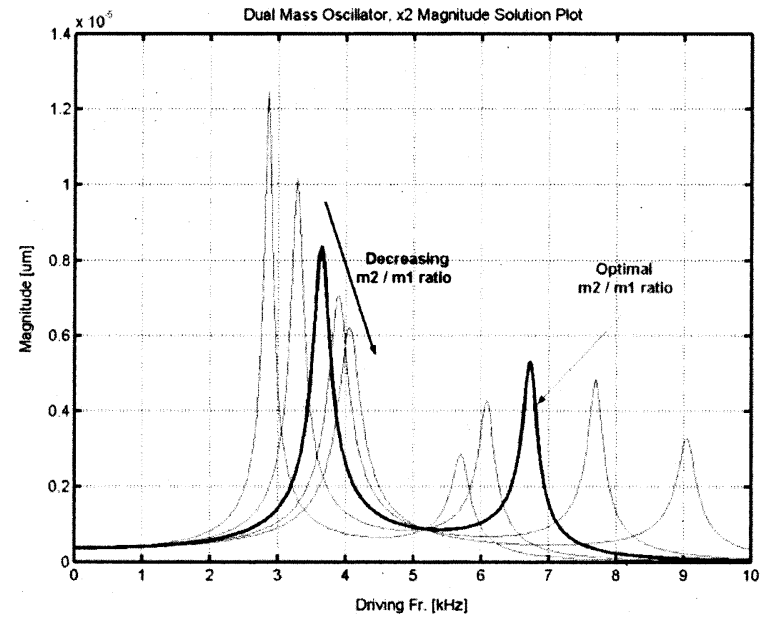

(a)

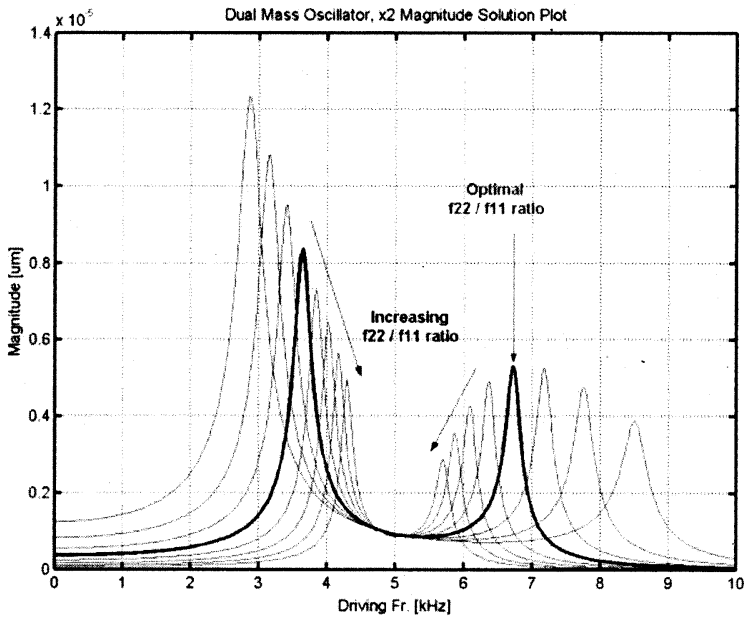

(b)

Fig. 10. Effect of (a) mass ratio $\mu_{x}=\left(m_{2}+m_{3}\right) / m_{1}$ variation and (b) frequency ratio $\gamma_{x}=\omega_{2 x} / \omega_{1 x}$ variation on drive direction response.

far enough, which imposes a minimum value of $\mu_{x}$. For a wide bandwidth, a large $\mu_{x}$ is required for large enough separation of the peaks; however, to prevent gain drop, the peak separation should be minimized [Fig. 10(a)]. 
The degree of mechanical amplification depends on the ratio of the resonance frequencies of the isolated active and passive mass-spring systems, namely $\gamma_{x}=\omega_{2 x} / \omega_{1 x}=\sqrt{k_{2 x} m_{1} / k_{1 x}\left(m_{2}+m_{3}\right)}$. The optimal frequency ratio $\gamma_{x}$ has to be determined such that $\gamma_{x}$ is high enough for high mechanical amplification, and high oscillation amplitudes of passive mass [Fig. 10(b)]. From the optimal values of $\omega_{1 x}$ and $\mu_{x}$, the drive direction spring constant $k_{1 x}$ of the active mass is obtained. Finally, the damping conditions of the overall device have to be checked to verify that damping values are in the region where the response gain in the antiresonance region is insensitive to damping variations (Fig. 11).

2) Sense Mode Parameters: The 2-DOF sense-direction oscillator is formed by $m_{2}$ and $m_{3}$, where $m_{3}$ acts as the vibration absorber to achieve large sense direction oscillation amplitudes due to mechanical amplification [Fig. 7(b)] . The objective of parameter optimization in the sense mode is to maximize $y_{3}$, which is the sense direction oscillation amplitude of the sensing element $m_{3}$.

The system is driven by the rotation-induced Coriolis forces $F_{c 2}=2 m_{2} \Omega_{z} \dot{x_{2}}$ and $F_{c 3}=2 m_{3} \Omega_{z} \dot{x_{2}}$ generated by $m_{2}$ and $m_{3}$, respectively. The dominant force exciting the 2-DOF sensedirection oscillator is $F_{c 2}$, since the mass of the active mass $m_{2}$ is significantly larger than the mass of the passive mass $m_{3}$. The equations of motion of the lumped mass-spring-damper model of the sense direction oscillator become

$$
\begin{aligned}
& m_{2} \ddot{y_{2}}+c_{2 y} \dot{y_{2}}+k_{2 y} y_{2}=k_{3 y}\left(y_{3}-y_{2}\right)+2 m_{2} \Omega_{z} \dot{x_{2}} \\
& m_{3} \ddot{y_{3}}+c_{3 y} \dot{y_{3}}+k_{3 y} y_{3}=k_{3 y} y_{2}+2 m_{3} \Omega_{z} \dot{x_{2}} .
\end{aligned}
$$

The response of the system to a constant-amplitude sinusoidal force is similar to that of the drive direction oscillator [Fig. 8(b)], with the resonant frequencies of the isolated active and passive mass-spring systems of $\omega_{2 y}=\sqrt{k_{2 y} / m_{2}}$ and $\omega_{3 y}=\sqrt{k_{3 y} / m_{3}}$, respectively. When the frequency of the sinusoidal Coriolis force is matched with the resonant frequency of the isolated passive mass-spring system, the passive mass $m_{3}$ achieves maximum dynamic amplification.

The most important advantage of decoupling the 2-DOF drive and sense direction oscillators is that the Coriolis force that excites the sensing element is not generated by the sensing element. Instead, $F_{c 2}=2 m_{2} \Omega_{z} \dot{x_{2}}$ generated by $m_{2}$ excites the active mass. The dynamics of the 2-DOF oscillator dictates that the mass of the passive mass $m_{3}$ has to be minimized in order to maximize its oscillation amplitude. Since the Coriolis Force $F_{c 3}=2 m_{3} \Omega_{z} \dot{x_{2}}$ generated by $m_{3}$ is not required to be large, the sensing element $m_{3}$ can be designed to be as small as the mechanical design requirements and fabrication parameters allow.

Similarly, the optimal mass ratio $\mu_{y}=m_{3} / m_{2}$ in the sense direction determining the mass of the active mass $m_{2}$ is selected to achieve insensitivity to damping variation, a wide response bandwidth and a large oscillation amplitude [18]. The optimal ratio of the resonance frequencies of the isolated active and passive mass-spring systems $\gamma_{y}=\omega_{3 y} / \omega_{2 y}=\sqrt{k_{3 y} m_{2} / k_{2 y} m_{3}}$ is also selected to maximize oscillation amplitudes of passive mass.

3) Overall 4-DOF System Parameters: The frequency response of both of the 2-DOF drive and sense direction oscil-

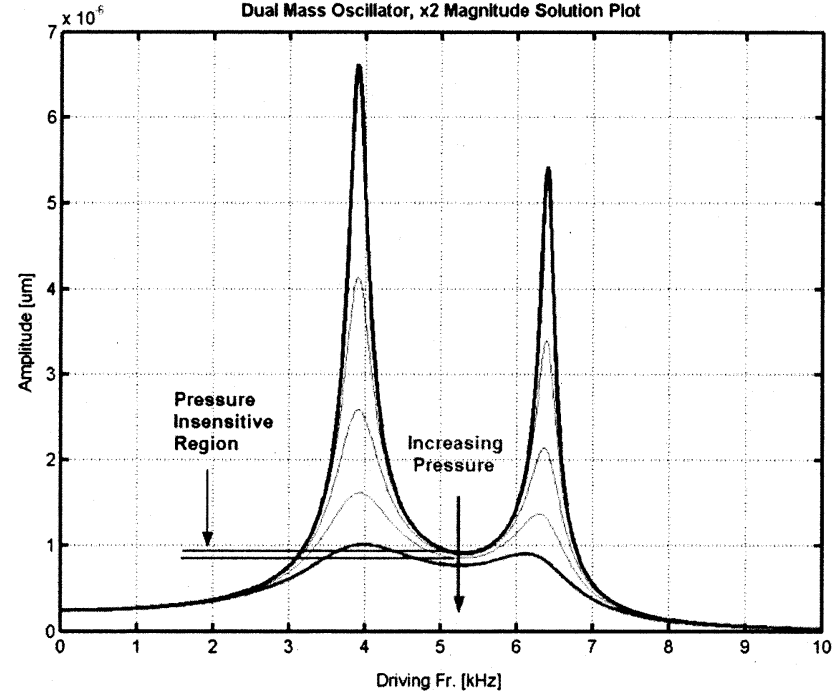

Fig. 11. Effect of damping on drive direction response. When the damping is under a critical value, the response in the flat region is insensitive to pressure changes.

lators have two resonant peaks and a flat region between the peaks. To achieve maximum robustness against fluctuations in the system parameters, both of the 2-DOF oscillators have to be operated in the flat region of their response curves. Since the Coriolis forces that drive the sense-direction oscillator are at the same frequency as the electrostatic forces exciting the drive-direction oscillator, the flat-region frequency band of the oscillators have to be overlapped, by designing the drive and sense anti-resonance frequencies to match. Thus, the requirement $\omega_{3 y}=\omega_{2 x}$, i.e., $\sqrt{k_{3 y} / m_{3}}=\sqrt{k_{2 x} /\left(m_{2}+m_{3}\right)}$, determines the optimal system parameters, together with the optimized ratios $\mu_{x}=\left(m_{2}+m_{3}\right) / m_{1}, \gamma_{x}=\omega_{2 x} / \omega_{1 x}, \mu_{y}=$ $m_{3} / m_{2}$, and $\gamma_{y}=\omega_{3 y} / \omega_{2 y}$. Since the flat regions have significantly wider bandwidths, they can be overlapped with sufficient precision without feedback control in the presence of imperfections, in contrast to the conventional gyroscopes.

Exciting the 2-DOF drive-direction oscillator at its anti-resonance frequency results in minimal oscillation amplitudes of the electrostatically driven mass [Fig. 8(a)]. Thus, by minimizing the travel distance of the actuators, higher actuation stability and linearity is achieved by means of mechanical amplification. Also, since the 2-DOF sense-direction oscillator is excited at its anti-resonance frequency, the sense-direction oscillation amplitude of $m_{2}$ is minimized [Fig. 8(b)]. This results in a minimal coupling between the oscillation modes, leading to reduced zero-rate drift of the gyroscope.

\section{CONCLUSION}

In this paper, a novel 4-DOF nonresonant micromachined gyroscope design concept is reported, which eliminates the mode-matching requirement, and minimizes instability and zero-rate drift due to mechanical coupling between the drive and sense modes. The proposed approach is based on forming mechanically decoupled 2-DOF drive-mode oscillator and 2-DOF sense-mode oscillator using three interconnected proof masses. The overall 4-DOF system utilizes dynamical 
amplification in the drive and sense directions to achieve large oscillation amplitudes without resonance resulting in increased bandwidth and reduced sensitivity to structural and thermal parameter fluctuations and damping changes, while mechanically decoupling the drive direction oscillations from the sense direction oscillations leads to improved robustness and long-term stability over the operating time of the device. Furthermore, employing three proof masses to form the decoupled oscillators allows the Coriolis force that excites the sensing element to be generated by a larger intermediate proof mass, resulting in larger Coriolis forces for increased sensor sensitivity. Thus, the design concept, which provides improved robustness and stability with minimal compromise in performance, is expected to relax control system requirements and tight fabrication and packaging tolerances.

\section{REFERENCES}

[1] N. Barbour and G. Schmidt, "Inertial sensor technology trends," IEEE Sensors J., vol. 1, pp. 332-339, Dec. 2001.

[2] N. Yazdi, F. Ayazi, and K. Najafi, "Micromachined inertial sensors," Proc. IEEE, vol. 86, pp. 1640-1658, Aug. 1998.

[3] W. A. Clark, R. T. Howe, and R. Horowitz, "Surface micromachined $z$-axis vibratory rate gyroscope," in Proc. Solid-State Sensor and Actuator Workshop, June 1994, pp. 199-202.

[4] A. Shkel, R. Horowitz, A. Seshia, S. Park, and R. T. Howe, "Dynamics and control of micromachined gyroscopes," in Amer. Contr. Conf., CA, 1999, pp. 2119-2124.

[5] S. Park and R. Horowitz, "Adaptive control for $z$-axis MEMS gyroscopes," in Amer. Contr. Conf., Arlington, VA, June 2001.

[6] R. P. Leland, "Adaptive tuning for vibrational gyroscopes," in Proc. IEEE Conf. Decision and Control, Orlando, FL, Dec. 2001.

[7] A. Shkel, R. T. Howe, and R. Horowitz, "Modeling and simulation of micromachined gyroscopes in the presence of imperfections," in Int. Conf. Modeling and Simulation of Microsystems, PR, 1999, pp. 605-608.

[8] W. A. Clark, "Micromachined Vibratory Rate Gyroscope," Ph.D. dissertation, Univ. of California, Berkeley, 1994.

[9] C. Acar and A. Shkel, "Four degrees-of-freedom micromachined gyroscopes," J. Modeling Simulation Microsyst., vol. 2, no. 1, pp. 71-82, 2001.

[10] J. A. Geen, "A path to low cost gyroscopy," in Solid-State Sensor and Actuator Workshop, Hilton-Head, SC, 1998, pp. 51-54.

[11] W. Geiger, W. U. Butt, A. Gaisser, J. Fretch, M. Braxmaier, T. Link, A. Kohne, P. Nommensen, H. Sandmaier, and W. Lang, "Decoupled microgyros and the design principle DAVED," IEEE Sensors J., pp. 170-173, 2001.

[12] Y. Mochida, M. Tamura, and K. Ohwada, "A micromachined vibrating rate gyroscope with independent beams for drive and detection modes," Sens. Actuators A, vol. 80, pp. 170-178, 2000.

[13] M. Niu, W. Xue, X. Wang, J. Xie, G. Yang, and W. Wang, "Design and characteristics of two-gimbals micro-gyroscopes fabricated with Quasi-LIGA process," in Int. Conf. Solid-State Sensor and Actuators, 1997, pp. 891-894.

[14] U. Breng, W. Guttman, P. Leinfelder, B. Ryrko, S. Zimmermann, D. Billep, T. Gessner, K. Hillner, and M. Weimer, "A bulk micromachined gyroscope based on coupled resonators," in Int. Conf. Solid-State Sensor and Actuators, Japan, 1999, pp. 1570-1573.

[15] H. T. Lim, J. W. Song, J. G. Lee, and Y. K. Kim, “A few deg/hr resolvable low noise lateral microgyroscope," in Proc. IEEE MEMS Conf., NV, 2002, pp. 627-630.

[16] S. E. Alper and T. Akin, "A symmetric surface micromachined gyroscope with decoupled oscillation modes," Sens. Actuators A, vol. 97, pp. 347-358, 2002
[17] Y. S. Hong, J. H. Lee, and S. H. Kim, "A laterally driven symmetric micro-resonator for gyroscopic applications," J. Micromech. Microeng., vol. 10 , pp. $452-458,2000$.

[18] C. W. Dyck, J. Allen, and R. Hueber, "Parallel plate electrostatic dual mass oscillator," Proc. SPIE, vol. 3876, pp. 198-209, 1999.

[19] W. C. Tang, "Electrostatic Comb-Drive for Resonant Sensor and Actuator Applications," Ph.D. dissertation, Univ. of California, Berkeley, 1990.

[20] B. E. Boser, "Electronics for micromachined inertial sensors," in Proc. Transducers, 1997.

[21] W. C. Young, Roark's Formulas for Stress and Strain. New York: McGraw-Hill, 1989, pp. 93-156.

[22] W. Kuehnel, "Modeling of the mechanical behavior of a differential capacitor acceleration sensor," Sens. Actuators A, vol. 48, pp. 101-108, 1995.

[23] T. Veijola and M. Turowski, "Compact damping models for laterally moving microsctructures," J. Microelectromech. Syst., vol. 10, no. 2, pp. 263-273, June 2001

[24] Y. H. Cho, B. M. Kwak, A. P. Pisano, and R. T. Howe, "Slide film damping in laterally driven microsctructures," Sens. Actuators A, vol. 40, pp. 31-39, 1994.

[25] C. Bourgeois, F. Parret, and A. Hoogerwerf, "Analytical modeling of squeeze-film damping in accelerometers," in Int. Conf. Solid-State Sensors and Actuators, Chicago, IL, June 1997, pp. 1117-1120.

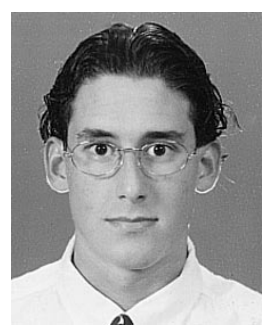

Cenk Acar was born in Turkey in 1977. He received the B.S. degree in mechanical engineering from Bogazici University, Turkey, and the M.S. degree in mechanical and aerospace engineering from the University of California, Irvine. He is currently pursuing the $\mathrm{Ph} . \mathrm{D}$. degree in mechanical and aerospace engineering at the University of California, Irvine.

His current research interests include design, modeling, fabrication, characterization, and control of microelectromechanical systems' inertial sensors.

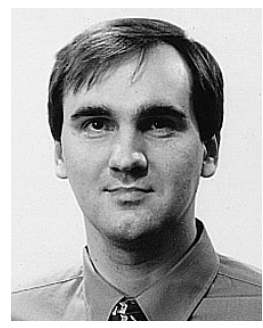

Andrei M. Shkel (A'98) received the Diploma (with excellence) in mechanics and mathematics from Lomonosov's Moscow State University, Moscow, Russia, in 1991 and the Ph.D. degree in mechanical engineering from the University of Wisconsin, Madison, in 1997.

$\mathrm{He}$ is an Assistant Professor in the Department of Mechanical and Aerospace Engineering, the Department of Electrical Engineering and Computer Sciences, and the Department of Biomedical Engineering, University of California (UC), Irvine, where he is also the Director of the UCI Micro-Systems Laboratory. From 1997 to 1999, he did his post-doctoral work at the Berkeley Sensor and Actuator Center (BSAC), UC, Berkeley. He then held research and consulting positions at several hi-tech and venture companies. His professional interests, reflected in over 50 publications and three U.S. patents, include solid-state sensors and actuators, MEMS-based neuroprosthetics, sensor-based intelligence, and control theory. He served as the Publications Chair of 2002 IEEE Sensors Conference, Vice General Chair and Publication Chair of 2003 IEEE Sensors, and member of technical committees of 2003 IEEE Sensors, 2001, 2002, 2003 SPIE, 2003 TMS, and 2001 ACC.

Dr. Shkel is an Associate Member of ASME and SPIE. He has served on a number of editorial boards, including Guest Editor for two special issues of the IEEE SENSORS JOURNAL, he was awarded the 2002 George E. Brown, Jr. Award, and he was the recipient of 2001 Fellowship of the Japanese Advanced Science Institute. 